\title{
Improving laparoscopic suturing skills
}

\author{
Maurice Anidjar, MD, PhD
}

See related article on page 22

A cquiring surgical skills is an important and very challenging step during residency. We remember the classic paradigm, "See one, do one, teach one," that applied to open surgery. The rapid and massive development of minimally invasive surgery (MIS) emphasized the urgent need to change this paradigm. Therefore, MIS skill laboratories have been created to transfer the surgical learning curve from the operating room (OR) to a more friendly, stress-free environment where residents can train without the limitations of OR time and can be assessed objectively through different physical and virtual reality simulators.

Most of the urology residency programs in North America now include an MIS curriculum where residents are assessed on a regular basis in dry laboratories before gaining access to animal laboratories and then to patients. Laparoscopic suturing remains a very advanced technical step that is the key to reconstructive urological procedures such as laparoscopic radical prostatectomy, laparoscopic pyeloplasty and laparoscopic partial nephrectomy.

Laparoscopic radical prostatectomy is a complex procedure requiring a difficult dissection directly related to familiarity with the male pelvic anatomy. Then a complex vesicourethral anastomosis in a deep and narrow pelvis should be performed with interrupted or running suture. Obviously this step could be mastered in a more expeditious way if we were able to train residents on a good task-specific model mimicking the surgical procedure.

The prospective randomized study by Sabbagh and colleagues ${ }^{1}$ comparing training on a task-specific lowfidelity bench suturing model versus basic laparoscopic suturing clearly demonstrates the superiority of the bench model. It therefore nicely proves that as difficult a laparoscopic task as vesico-urethral anastomosis could be learned and mastered outside of the OR with objective scoring assessment. The authors should therefore be commended for this achievement.

Associate Member, Division of Urology, Department of Surgery, Royal Victoria Hospital, McGill University Health Centre, Montréal, Que.

Competing interests: None declared.

\section{Reference}

1. Sabbagh R, Chatteriee $S$, Chawla A, et al. Task-specific bench model training versus basic laparoscopic skills training for laparoscopic radical prostatectomy: a randomized controlled study. Can Urol Assoc J 2009; 1:22-30.

Correspondence: Dr. Maurice Anidjar, Division of Urology, McGill University Health Centre, Royal Victoria Hospital, 687 Pine Ave. West, S6.88, Montréal QC H3A 1A1; maurice.anidjar@muhc.mcgill.ca. 